\title{
Cuing the dimension of a distractor: Verbal cues of target identity also benefit same-dimension distractor singletons
}

\author{
MARTIJN MEETER and JAN THEEUWES \\ Vrije Universiteit Amsterdam, Amsterdam, The Netherlands
}

\begin{abstract}
Cuing the identity of an upcoming target speeds its detection. This effect is generally assumed to operate on the level of the target dimension, not of its feature identity. Here, we investigated whether that is the case, in a design in which preparing for a cued dimension would incur costs as well as benefits. Participants searched for targets that could be defined on several dimensions, but were also presented with distractors that were defined on the same dimensions. Cuing the identity of an upcoming target increased the effect of distractors defined on the same dimension as the target. This suggests that cuing a target's identity has effects that operate at least partly at the level of the target dimension.
\end{abstract}

We spend a lot of time searching for things, such as a traffic sign at a busy crossroad, our car in the parking lot, or one of our kids in a busy shopping mall. Common sense suggests that knowing what we are looking for helps this search process. For example, when searching for one of our lost kids in the daycare center, we try to remember what the child was wearing that day so that the exact color of the sweater may guide our search process.

Almost all theories of visual search assume that preknowledge of specific properties of the target will generate top-down activation that can guide the search process (Müller, Heller, \& Ziegler, 1995; Müller, Reimann, \& Krummenacher, 2003; Treisman, 1991; Wolfe, 1994, 1998; Wolfe, Butcher, Lee, \& Hyle, 2003). Top-down activation refers to the extent to which an item matches the current attentional set. Even though there is general agreement that preknowledge may guide search, there is some controversy about the level at which top-down guidance operates. Some theories maintain that top-down attentional set can only operate on specific dimensions, such as color, shape, and luminance (see Müller et al., 1995; Müller et al., 2003; Treisman, 1991). Others (e.g., Wolfe et al., 2003) suggest that top-down set can operate both on a stimulus dimension as well as on specific levels within such a dimension (e.g., by directing attention to the color red or to a left-tilted line segment). Finally, some models of attention make no reference to any stimulus dimension, but claim that top-down activation may directly act upon preattentive features (e.g., Navalpakkam \& Itti, 2005).

This study was supported by Grant 402-01-630 from the Dutch National Science Foundation (NWO) to the second author. Correspondence concerning this article should be addressed to M. Meeter, Department of Cognitive Psychology, Vrije Universiteit Amsterdam, Van der Boechorststraat 1, 1081 BT Amsterdam, The Netherlands (e-mail: m@meeter.nl).
When confronted with a display in which one element is unique in a basic visual dimension (such as a red element surrounded by green elements), one is able to immediately detect this element without effort. Elements that pop out from the display are referred to as feature singletons, or simply singletons. It is generally assumed that when confronted with such a display, it is segmented in parallel, as a set of basic stimulus attributes in different dimensionspecific modules (such as color, orientation, etc.). When in a singleton search task the target may be defined on one of two or more dimensions, performance typically suffers relative to the situation with only one possible target dimension (Found \& Müller, 1996; Müller et al., 1995; Treisman, 1988). In such experiments, participants have to detect the presence or absence of a singleton target among homogeneous filler elements, and the target can be defined, for example, by a unique color or orientation.

Although knowing the actual feature value of the target (e.g., whether it was blue or red) hardly speeded search in her experiment, Treisman (1988) showed that knowing the dimension of the target (whether it was a unique color or shape) speeded search by about $100 \mathrm{msec}$. Treisman (1988) suggested that there is no top-down selectivity within dimensions, but across dimensions, knowing the dimension in which the target will be presented speeds up search significantly. In her study, knowledge of target identity was manipulated by presenting different targets either in different blocks or by randomly intermixing them. This design confounded target knowledge with the automatic priming effects that have also been observed in visual search (Müller et al., 2003). A way to disentangle expectancy and priming effects is to randomly intermix different targets but to inform participants of the dimension of the upcoming target with a symbolic cue. Such cuing also speeds target detection (Müller et al., 2003; Theeuwes, Reimann, \& Mortier, in press): If a color tar- 
get is preceded by a cue consisting of the word "color," it is found faster than if no cue is provided or the word "orientation" is incorrectly given as a cue.

In agreement with Treisman (1988), Müller et al. (2003) suggested that effects of the cue operate at the level of the target dimension, not at the level of its feature value. In their Experiment 3, they cued target features instead of dimensions. For example, green targets were cued with the word "green" instead of with the word "color." When a cue for a particular feature was followed by a target that was defined by another feature on the same dimension (e.g., the cue "red" followed by a green target), performance was still better than when no feature was cued. Moreover, for the orientation dimension, the difference between a correct feature cue and one that cued the correct dimension but the wrong feature was not reliable (e.g., a right oblique target was detected equally fast after the cues "left oblique" and "right oblique"). If one sees the benefit of cuing as resulting from preparation, it would seem that participants prepare for a target dimension, not a target feature.

Although these experiments suggest that cuing normally operates at the level of dimensions, it is unclear to what extent preparation for target dimensions (and not target features) is obligatory. In other words, is it impossible to prepare for just the target feature? Or is it simply more beneficial to prepare for a whole dimension? Indeed, if there are no obvious costs associated with preparing for all features of a particular dimension, as opposed to preparing only for one of its features, one may simply not see feature-specific preparation in the data. Observers could simply prepare for all features of a dimension, because preparation for a whole dimension is just as easy as preparing for just one feature.

A design in which preparing for all features in a given dimension would incur costs as well as benefits would enable an assessment of whether preparing for a dimension instead of for a feature is obligatory. A manipulation that would ensure costs as well as benefits would be to include distractor singletons that could be defined on either the cued or the uncued dimension. If distractor singletons slow responses more when their dimension is cued than when it is not, one can conclude that preparing for a dimension, as opposed to preparing for a feature, is obligatory.

\section{METHOD}

To investigate the effects of dimensional cues on distractor singletons, the participants had to find a target that could be defined on one of two dimensions, while disregarding possible distractor singletons that were defined on the same dimensions. Targets could either be green or oblique gray stripes, both of which popped out against a background of vertical gray elements (see Figure 1). Distractor singletons were either red or horizontal.

On some trials, the search display was preceded by a verbal cue that predicted the identity of the target with $100 \%$ validity (e.g., after the cue "color," the participants knew that a target would be either green or absent). We investigated the effects of these cues with distractor singletons. If cuing the identity of a target elicits preparation for the target dimension, distractor singletons should have a larger effect on reaction times (RTs) when they are defined on the dimension corresponding to the cue than when they are defined on the uncued dimension (e.g., red distractor singletons should slow responses more after a color cue than after an orientation cue). If cuing elicits preparation for the target feature alone, cuing should not change the effect of distractor singletons.

It is possible, though not very likely, that the observers could have prepared for a dimension after a dimension cue and for a feature after a feature cue. Therefore, we conducted two experiments, $1 \mathrm{~A}$ and $1 \mathrm{~B}$. In Experiment 1A, the observers received cues that indicated the target's dimension, and in Experiment 1B they received cues that indicated the target's feature.

Cuing the distractor singleton is confounded with cuing the target. For example, a color cue may lead to better processing of red distractor singletons, but it also enhances processing of green targets. To disentangle the effects of cues on distractor singletons from the effects of cues on targets, we only investigated the effect of cues on distractor singletons in trials in which the target would equally benefit from color and orientation cues. There were two categories of trials for which this was the case: target-absent trials and trials on which the target was an oblique green stripe (i.e., a target that popped out on both dimensions). Following Krummenacher, Müller, and Heller (2001), we called the oblique green targets redundant targets. On trials with such redundant targets, both dimension cues cued the target, but cues could match or not match the distractor singleton dimension. This allowed a clean measure of the effect of cuing on distractor singletons. If cues affected distractor singletons, there would be an interaction between the dimension that was cued and that of the distractor singleton. In particular, RTs for red distractor singletons should increase more after a color cue than after an orientation cue, but the opposite should be true for horizontal distractor singletons. On target-absent trials, cuing could not affect processing of a target that was not there, and we therefore tested the same hypothesis for those trials.

Although our hypotheses were tested only on trials with redundant targets and on target-absent trials, we also included trials with green targets and oblique targets to give the participants an incentive to heed the cues. If all targets had been green and oblique, a cue would not have been informative about target identity.

In summary, we set out to test three hypotheses. First, cuing should reduce RTs. Second, for redundant-target trials, there should be an interaction between cue identity and distractor identity, in that RTs should be slowed more when cue and distractor identities match. Third, the same interaction should be present for targetabsent trials.

\section{Participants and Apparatus}

Eight observers participated in Experiment 1A for a small monetary compensation ( 4 men and 4 women, with a mean age of 21). Sixteen observers participated in Experiment 1B (10 women and 6 men, mean age 24). All had normal or corrected-to-normal eyesight and normal color vision. They were seated in a dimly lit room at a distance of approximately $70 \mathrm{~cm}$ from a 21 -in. monitor.

\section{Stimuli}

Search displays consisted of $13 \times 9$ line elements, covering an area of $8.2^{\circ} \times 7.4^{\circ}$ of the visual field (see Figure 1). Targets and distractor singletons were present on half of the trials, and target presence and distractor presence were varied independently of one another (e.g., a target and distractor singleton were both present on $50 \% \times 50 \%=25 \%$ of trials). Filler line elements were vertical and gray, with a height of approximately $1^{\circ}$ and a width of $0.16^{\circ}$. Targets were the same size as the filler elements and could be a green color singleton, a right oblique orientation singleton $\left(30^{\circ}\right.$ rotation $)$, or, for redundant targets, a green oblique line element that was both a color and orientation singleton. Distractor singletons were either a red (color singleton) or a horizontal (orientation singleton) line of 


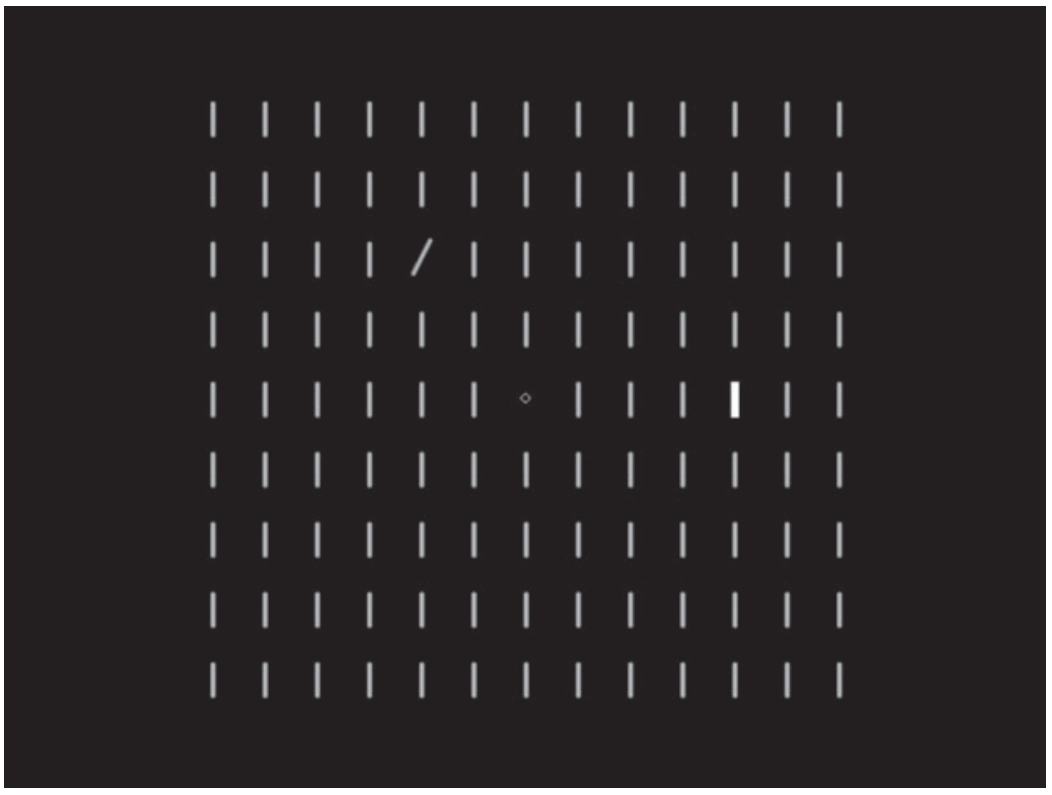

Figure 1. Example of a search display. Here, an oblique target (upper left) is accompanied by a color distractor singleton (middle right).

the same size as the filler elements. If present, targets and distractor singletons replaced one filler element in one of six positions on an imaginary circle $5.5^{\circ}$ in diameter around the fixation point. The locations of targets and distractor singletons were randomized, with the proviso that both could not be at the same location. In the display shown in Figure 1, an orientation target in the upper left quadrant is accompanied by a color distractor singleton to the right of fixation.

\section{Procedure}

We used a factorial design with five factors: target presence, target identity, distractor presence, distractor identity, and cuing. A sixth factor, cue identity, covaried with target identity. Trials were organized in six blocks of 72 trials, preceded by 40 practice trials. In four blocks, trials were preceded by a symbolic cue. In Experiment $1 \mathrm{~A}$, these were dimension cues, either the word "color" or the word "oblique."1 In Experiment 1B, they were feature cues denoting the target feature "green" or "right" (for right oblique). Both sets of cues were equally predictive, since only one target could appear after either a dimension or a feature cue. In the two remaining blocks, no cue was given. Instead, a star preceded the search display. Order of presentation of the blocks was randomized. Cues were $100 \%$ valid, always predicting the dimension of the target if there was one. Target presence, target identity, distractor presence, and distractor identity were all randomly intermixed.

Trials began with a 400-msec presentation of a small fixation circle. Then the cue (or a star in no-cue trials) was presented for $750 \mathrm{msec}$, followed by another 750 -msec presentation of the fixation circle. Then the search display was presented until the participant responded. They were required to respond in target-absent trials with the $z$ key, in target-present trials with the $m$ key. Errors were followed by a short beep, and every 24 trials the participants were informed of their mean RT and error rate.

\section{RESULTS}

\section{Reaction Times}

Trials with RTs longer than 1,200 msec, which were less than $1 \%$ of the total in both experiments, were ex- cluded from the analysis. On average, participants made errors on $5.1 \%$ of trials in Experiment $1 \mathrm{~A}$ and $5.4 \%$ of trials in Experiment 1B, and these trials were also excluded from RT analyses.

We first performed an ANOVA on mean RTs with target type, distractor presence, and cuing as factors (see Table 1). Degrees of freedom were Greenhouse-Geisser corrected when the assumption of sphericity was violated. In Experiment 1A, main effects of target type $[F(3,21)=$ $19.88, p<.001]$ and distractor presence $[F(2,14)=$ $13.69, p=.001]$ occurred, with distractor singletons slowing RTs by about $30 \mathrm{msec}$. There was also a trend toward faster responding on cued trials than on uncued trials $[F(1,7)=3.68, p=.096$; tested with a one-sided $t$ test, this effect was significant, $p<.048]$. The same main effects were present in Experiment 1B [target type, $F(1.45,21.78)=18.71, p<.001$; distractor presence, $F(2,30)=10.31, p<.001$, with distractor singletons slowing RTs by around $30 \mathrm{msec}]$, as well as a main effect of cuing $[F(1,15)=5.31, p=.036$; cued trials were faster than uncued trials]. In Experiment 1A, none of the interactions were significant, but in Experiment 1B, there was an interaction between target type and distractor presence $[F(6,90)=5.16, p<.001]$, with larger distractor effects in target-absent than in target-present trials.

\section{Errors}

To determine whether the proportion of errors was influenced by any of the independent variables, we transformed error proportions to a form suitable for ANOVAs ${ }^{2}$ (Winer, Brown, \& Michels, 1991) and performed the same omnibus analysis we used for RTs.

In Experiment 1A, more errors were made when there was a distractor singleton than when there was none 
Table 1

Mean Reaction Times (RTs, in Milliseconds) and Proportions of Errors for All Conditions Defined by Target Type, Distractor Singleton Identity, and Cue

\begin{tabular}{|c|c|c|c|c|c|c|c|c|c|c|c|c|}
\hline Analysis and Cue & None & Color & Oblique & None & Color & Oblique & None & Color & Oblique & None & Color & Oblique \\
\hline \multicolumn{13}{|c|}{ Experiment 1} \\
\hline \multicolumn{13}{|l|}{ RTs } \\
\hline No cue & 512 & 573 & 572 & 464 & 487 & 473 & 516 & 539 & 569 & 500 & 502 & 496 \\
\hline "Color" & 485 & 548 & 523 & 447 & 475 & 436 & 491 & 524 & 513 & & & \\
\hline \multicolumn{13}{|l|}{ Errors } \\
\hline No cue & .017 & .044 & .058 & .010 & .030 & .036 & .085 & .104 & .016 & .129 & .151 & .085 \\
\hline "Color" & .020 & .049 & .029 & .040 & .000 & .041 & .093 & .140 & .063 & & & \\
\hline "Oblique" & .024 & .029 & .047 & .014 & .036 & .101 & & & & .099 & .071 & .024 \\
\hline \multicolumn{13}{|c|}{ Experiment 2} \\
\hline \multicolumn{13}{|l|}{ RTs } \\
\hline \multicolumn{13}{|l|}{ Errors } \\
\hline No cue & .017 & .030 & .036 & .026 & .026 & .031 & .109 & .078 & .078 & .120 & .094 & .073 \\
\hline "Green" & .019 & .054 & .056 & .073 & .036 & .021 & .133 & .089 & .086 & & & \\
\hline "Right" & .030 & .033 & .063 & .047 & .036 & .031 & & & & .073 & .107 & .055 \\
\hline
\end{tabular}

$[F(2,14)=15.94, p<.001]$, and more errors were made on target-absent than on target-present trials $[F(3,21)=$ 25.67, $p<.001]$. Moreover, fewer errors were made when the target dimension was cued $[F(1,7)=9.39, p=.018]$. There was a significant interaction between target type and distractor presence $[F(6,42)=2.48, p=.038]$, with distractor presence leading to more errors on all types of trials, but less so on target-absent trials. Moreover, there was an interaction between cuing and target type $[F(3,21)=7.38, p=.001]$, with most errors being made with uncued orientation targets.

In Experiment 1B, there was a main effect of target type $[F(2,45)=36.223, p<.001]$ but not of distractor identity $(F<1)$ or cuing $[F(1,15)=2.70, p>.1]$. There were interactions between target type and distractor presence $[F(6,90)=3.19, p=.007]$ and target type and cuing $[F(3,45)=12.69, p<.001]$. Errors were made more often on target-present than on target-absent trials; they were more frequent with cuing in target-absent trials, but less frequent with cuing in target-present trials.

In both experiments, errors thus generally varied in the same direction as RTs. Speed-accuracy trade-offs are thus unlikely to have played a role in our RT analyses.

\section{Interaction Between Cuing and Distractor Dimension}

The above analyses did not include cue identity as a factor. To investigate the effect of cue identity on responses to distractor singletons, we investigated target-absent trials and trials with redundant targets.

Figure 2 shows the distractor effect (distractor singleton present - distractor singleton absent) for both kinds of trial in Experiment 1A, separately for color and orientation distractor singletons and the two cues. For redundant- target trials, the interaction between cuing and distractor identity was significant $[F(1,7)=10.45, p=.014]$. For target-absent trials, there was a trend toward an interaction in the right direction $[F(1,7)=4.13, p=.08]$. As Figure 2 shows, in both conditions distractor effects were larger when the distractor singleton was defined on the cued rather than the uncued dimension. Thus, horizontal distractor singletons slowed responses more after an "oblique" cue than after a "color" cue, and the opposite was true for red distractor singletons. In both conditions, there were no main effects of either cue or distractor identity.

Figure 3 shows distractor effects (distractor singleton present - distractor singleton absent) in Experiment 1B, separately for color and orientation distractor singletons and the two cues. For redundant-target trials, the interaction between cuing and distractor identity was again significant $[F(1,15)=5.36, p=.035]$. The results were in the right direction for target-absent trials, although the interaction there was not significant $[F(1,15)=2.48, p=$ .13]. As Figure 3 shows, in both conditions distractor effects were larger when the distractor singleton was defined on the cued rather than the uncued dimension. Thus, horizontal distractor singletons slowed responses more after a "right" cue than after a "green" cue, and red distractor singletons slowed responses more after a "green" cue than after a "right" cue.

We repeated the same analysis with transformed error proportions. We found no interaction between cue and distractor identities on either target-absent $[F(1,7)=1.15$, $p=.33$ ] or redundant-target $(F<1)$ trials in Experiment 1A (see Figure 2). The same was true for redundant targets $(F<1)$ in Experiment 1B, although there was a strong trend in the right direction for target-absent trials $[F(1,15)=4.23, p=.06$; see Figure 3$]$. In all other condi- 

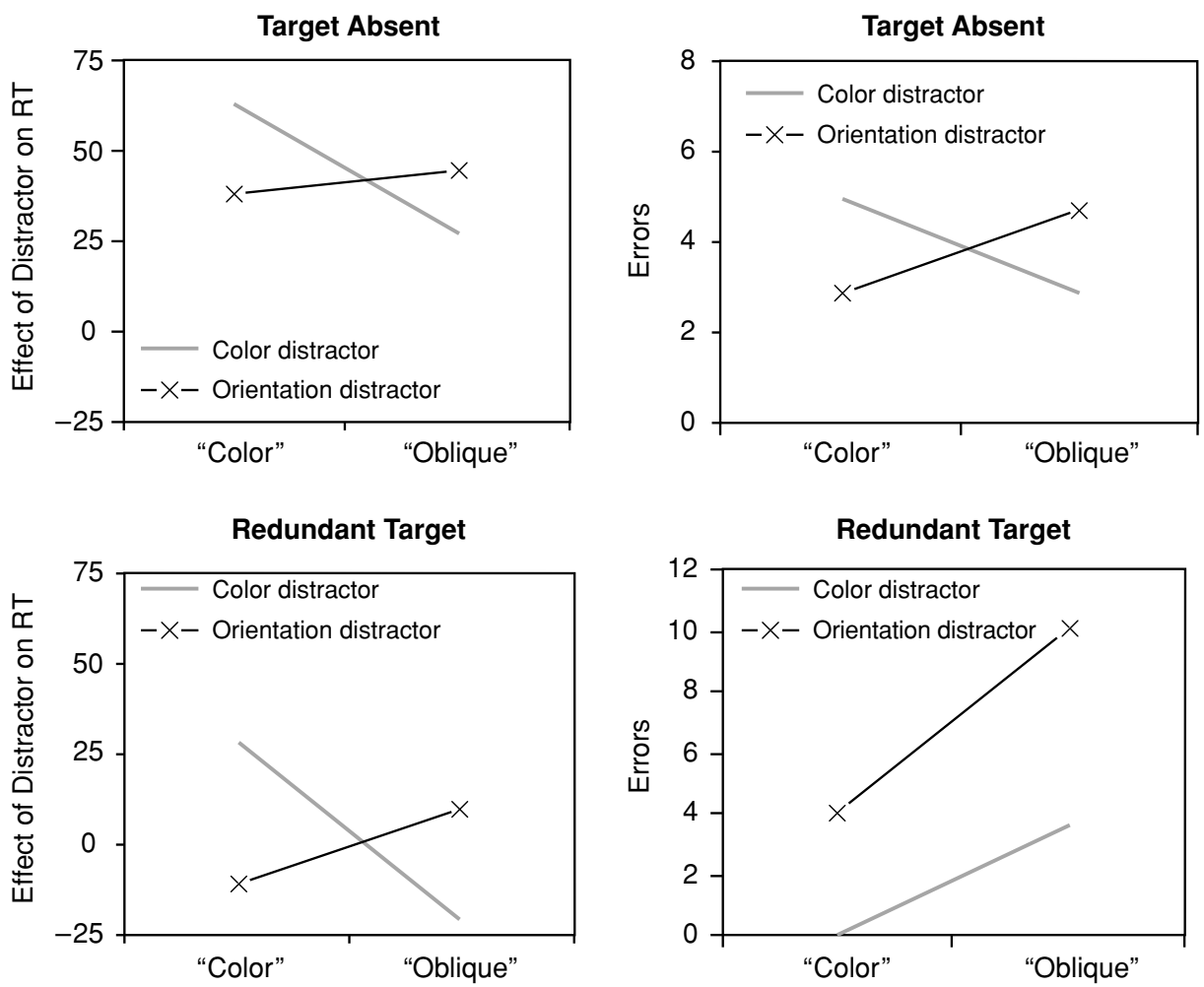

Figure 2. Slowing with respect to the no-distractor baseline and error percentages in Experiment 1 for target-absent trials and trials with redundant targets, split out for distractor identity (color or orientation distractor singleton) and cue identity ("color" or "oblique").

tions, as well, the pattern for mean error proportions was the same as that for RTs in both experiments, excluding speed-accuracy trade-offs.

Inspection of the redundant-target results in Figure 3 suggests that cuing in Experiment 1B may have had less of an effect on color than on orientation distractor singletons. We tested this formally by computing an "absolute cuing effect" (as the absolute value [RT after "color" cue - RT after "oblique" cue]) for both red and horizontal distractors, and testing whether they differed. This was not the case $(t<1)$.

\section{DISCUSSION}

Our results show that cuing the identity of the target made detection of the target faster and led to fewer errors. Cuing also had an influence on the processing of distractor singletons: When distractor singletons were defined on the dimension corresponding to the cue, they slowed responses more than when they were defined on a different dimension. This suggests that cuing leads to preparation that makes observers more sensitive to all stimuli defined on the dimension corresponding to the cue. For example, cuing a color target may lead to preparation benefiting all color singletons, not only those in the color that defines targets (e.g., green). This was the case regardless of whether the cue predicted the target dimension
(Experiment 1A) or the target feature (Experiment 1B). Since preparing for the target dimension instead of the target feature carried costs in the present experiments, we conclude that participants may not be able to restrict their preparation to a specific target feature. Instead, preparation for a target may occur by necessity at least partly at the level of the dimension.

Our results could be taken to suggest that verbal cues only work at the dimension level, but such a strong conclusion would be difficult to reconcile with findings in conjunctive search. Several findings suggest that participants can effectively ignore sets of nontargets on the basis of a feature and search only among elements of a certain color (Kaptein, Theeuwes, \& van der Heijden, 1995) or letters with a certain identity (Egeth, Virzi, \& Garbart, 1984). It is possible that in these studies, efficient use of features was not the result of top-down knowledge but of bottomup priming (e.g., Maljkovic \& Nakayama, 1994, 2000), since the feature that guided selection usually remained constant over a number of trials. Another interpretation fitting both findings is that attentional selection can be based on a feature, but that during preparation attention "leaks" to other features defined on the same dimension.

Several theories suggest that verbal cues such as the ones used here elicit top-down guidance that works at the dimension level (Müller et al., 1995; Müller et al., 2003; Treisman, 1991), but none of these theories explains why 
Target Absent

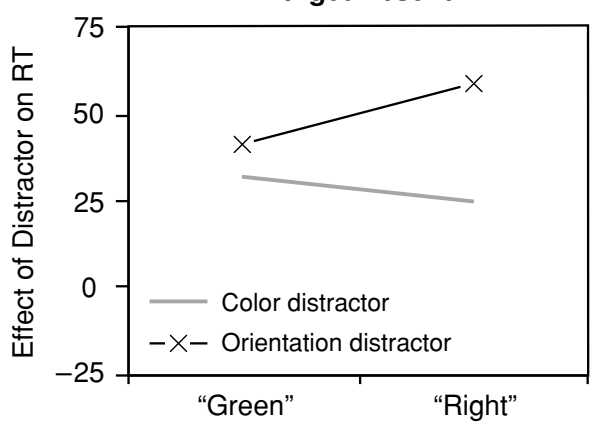

Redundant Target

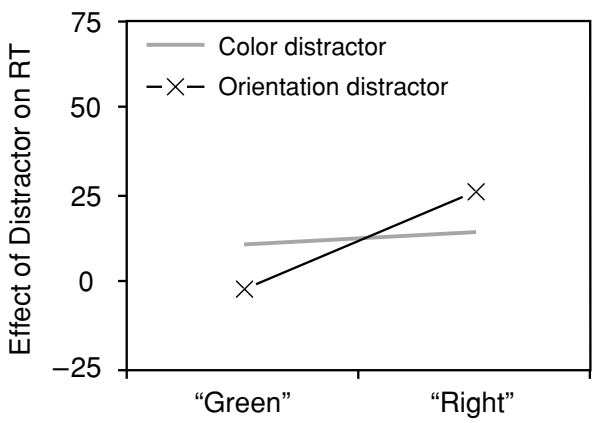

Target Absent

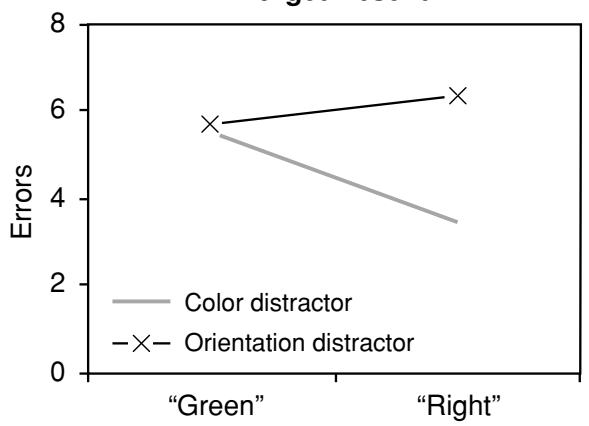

Redundant Target

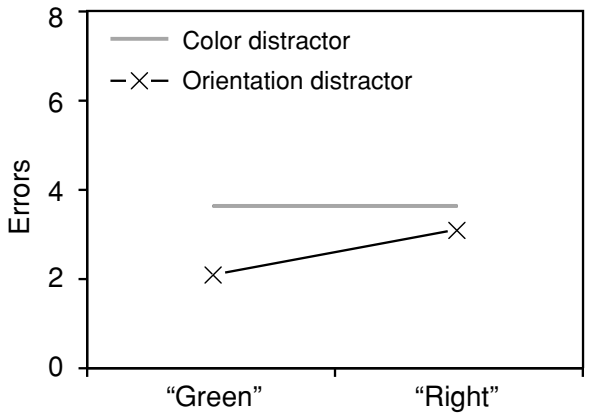

Figure 3. Slowing with respect to the no-distractor baseline and error percentages in Experiment 2 for target-absent trials and trials with redundant targets, split out for distractor identity (color or orientation distractor singleton) and cue identity ("green" or "right").

top-down guidance should operate at the level of dimensions instead of at the level of specific feature maps. Evidence from cognitive neuroscience has suggested one answer: Different dimensions of visual stimuli are processed in different areas of the brain. When attention is directed toward visual dimensions, activation in commensurate brain areas has been found in advance of stimuli, reflecting top-down preparation (Liu, Slotnick, Serences, \& Yantis, 2003; Shulman, d'Avossa, Tansy, \& Corbetta, 2002). Within brain areas that analyze visual dimensions, neurons sensitive to different features are generally intermixed (Eysel, Muche, \& Worgotter, 1988; Roe \& Ts'o, 1995). It is possible that although top-down mechanisms can activate dimension-specific processing areas, they are spatially too coarse to support preparation for specific features.

\section{REFERENCES}

Egeth, H. E., Virzi, R. A., \& Garbart, H. (1984). Searching for conjunctively defined targets. Journal of Experimental Psychology: Human Perception \& Performance, 10, 32-39.

Eysel, U. T., Muche, T., \& Worgotter, F. (1988). Lateral interactions at direction-selective striate neurones in the cat demonstrated by local cortical inactivation. Journal of Physiology, 399, 657-675.

Found, A., \& MüLler, H. J. (1996). Searching for unknown feature targets on more than one dimension: Investigating a "dimensionweighting" account. Perception \& Psychophysics, 58, 88-101.

Kaptein, N. A., Theeuwes, J., \& van der Heijden, A. H. C. (1995). Search for a conjunctively defined target can be selectively limited to a color-defined subset of elements. Journal of Experimental Psychology: Human Perception \& Performance, 21, 1053-1069.

Krummenacher, J., Müller, H. J., \& Heller, D. (2001). Visual search for dimensionally redundant pop-out targets: Evidence for parallel-coactive processing of dimensions. Perception \& Psychophysics, 63, 901-917.

LiU, T., SLotNick, S. D., Serences, J. T., \& Yantis, S. (2003). Cortical mechanisms of feature-based attentional control. Cerebral Cortex, 13, 1334-1343.

Maljkovic, V., \& Nakayama, K. (1994). Priming of pop-out: I. Role of features. Memory \& Cognition, 22, 657-672.

Maljkovic, V., \& Nakayama, K. (2000). Priming of popout: III. A short-term implicit memory system beneficial for rapid target selection. Visual Cognition, 7, 571-595.

Müller, H. J., Heller, D., \& Ziegler, J. (1995). Visual search for singleton feature targets within and across feature dimensions. Perception \& Psychophysics, 57, 1-17.

Müller, H. J., Reimann, B., \& Krummenacher, J. (2003). Visual search for singleton feature targets across dimensions: Stimulusand expectancy-driven effects in dimensional weighting. Journal of Experimental Psychology: Human Perception \& Performance, 29, 1021-1035.

NavalpakKam, V., \& ItTi, L. (2005). Modeling the influence of task on attention. Vision Research, 45, 205-231.

RoE, A. W., \& Ts'o, D. Y. (1995). Visual topography in primate V2: Multiple representation across functional stripes. Journal of Neuroscience, 15, 3689-3715.

Shulman, G. L., D’Avossa, G., Tansy, A. P., \& Corbetta, M. (2002). Two attentional processes in the parietal lobe. Cerebral Cortex, 12, 1124-1131.

Theeuwes, J., Reimann, B., \& Mortier, K. (in press). Visual search for featural singletons: No top-down modulation, only bottom-up priming. Visual Cognition.

Treisman, A. (1988). Features and objects: The Fourteenth Bartlett Me- 
morial Lecture. Quarterly Journal of Experimental Psychology, 40A, 201-237.

Treisman, A. (1991). Search, similarity, and integration of features between and within dimensions. Journal of Experimental Psychology: Human Perception \& Performance, 17, 652-676.

Winer, B. J., Brown, D. R., \& Michels, K. M. (1991). Statistical principles in experimental design. New York: McGraw-Hill.

WoLfE, J. M. (1994). Guided Search 2.0: A revised model of visual search. Psychonomic Bulletin \& Review, 1, 202-238.

Wolfe, J. M. (1998). Visual search. In H. Pashler (Ed.), Attention (pp. 13-73). Hove, U.K.: Psychology Press.

Wolfe, J. M., Butcher, S. J., LeE, C., \& Hyle, M. (2003). Changing your mind: On the contributions of top-down and bottom-up guidance in visual search for feature singletons. Journal of Experimental Psychology: Human Perception \& Performance, 29, 483-502.

\section{NOTES}

1. This was done because that word is short and common in Dutch, and the word "orientation" is long and has low frequency, and therefore is difficult to process. The word "oblique" does not cover all features of the orientation dimension (i.e., not straight lines). However, the results clearly show that "oblique" worked as a dimension cue and not as a feature cue.

2. $x=2 \arcsin \sqrt{ }(p+1 / 2 n)$, where $x$ is the transformed variable, $p$ is the proportion, and $n$ is the number of observations underlying the proportion.

(Manuscript received November 9, 2004; revision accepted for publication June 1, 2005.) 